\title{
Melanomfrüherkennung durch sequentielle digitale Dermatoskopie
}

\author{
Early Detection of Melanoma Through Digital Epiluminescence \\ Microscopy (DELM)
}

Autor

Institut

\section{H. A. Haenssle}

Abteilung Dermatologie und Venerologie, Georg-August-Universität Göttingen

\section{Bibliografie}

Dol $10.1055 / \mathrm{s}-0028-1119440$

Akt Dermatol 2009; 35:

75-78 @ Georg Thieme

Verlag KG Stuttgart · New York ISSN 0340-2541

Korrespondenzadresse Dr. med. Holger A. Haenssle Abteilung Dermatologie und Venerologie Georg-August-Universität von-Siebold-Str. 3 37075 Göttingen h.haenssle@med.unigoettingen.de

\section{Zusammenfassung}

Für Patienten mit einer hohen Anzahl atypischer Nävi sowie einer positiven Eigen- und/oder Familienanamnese für Melanome liegt ein erhöhtes Melanomerkrankungsrisiko vor. Im Rahmen einer Früherkennungsstudie wurden 530 Patienten anhand von Anzahl/Phänotyp der Nävi und der Eigen- und Familienanamnese für Melanome in Risikogruppen eingeteilt und in regelmäßigen Abständen (alle 3-12 Monate) klinisch, auflichtmikroskopisch und digital-auflichtmikroskopisch untersucht. Der durchschnittliche Beobachtungszeitraum betrugt 32,2 Monate. Bei Patientenerstvorstellung konnten 37 kutane Melanome und über den folgenden Beobachtungszeitraum weitere 53 Melanome identifiziert werden. 18 der Melanome des Beobachtungszeitraumes

\section{Hintergrund \\ $\nabla$}

Für Patienten mit einer hohen Anzahl atypischer Nävi sowie einer positiven Eigen- und/oder Familienanamnese für Melanome liegt ein deutlich erhöhtes Melanomerkrankungsrisiko vor ( $\bullet$ Abb. 1). In dieser Hochrisiko-Patientengruppe bestehen aufgrund der Vielzahl atypischer Pigmentläsionen besondere Schwierigkeiten, maligne Melanome frühzeitig zu erkennen. Nachdem Techniken für eine digitale Speicherung auflichtmikroskopischer Bilder zur Verfügung standen, wurde 1997 an der Universitäts-Hautklinik Göttingen eine klinische Studie zur Bedeutung der sequentiellen digitalen Auflichtmikroskopie für die langfristige Kontrolle atypischer Pigmentläsionen von Patienten mit erhöhtem Melanomrisiko entworfen. Nachdem das Studiendesign und die Programmierung einer geeigneten Datenbank abgeschlossen waren, konnte die Niedersächsische Krebsgesellschaft e.V. als Sponsor der Studie gewonnen werden. Im Jahr 2006 wurde das Pro- wurden ausschließlich aufgrund der Analyse der digitalen auflichtmikroskopischen Verlaufsbilder entdeckt. In diesen Fällen wurden geringgradige Verlaufsveränderungen festgestellt, ohne dass nach Analyse mittels diagnostischer Algorithmen der Verdacht für ein Melanom vorlag. Alle identifizierten Melanome konnten in einer frühen Progressionsphase (Breslow $<1 \mathrm{~mm}$ ) einer operativen Therapie zugeführt werden. Eine regelmäßige klinische, auflichtmikroskopische und besonders digital auflichtmikroskopische Verlaufsuntersuchung von Patienten mit einem erhöhten Melanomerkrankungsrisiko ermöglichte die frühzeitige Identifizierung von malignen Melanomen. Die digitale sequentielle Dermatoskopie steigerte zudem durch die Detektion zusätzlicher Melanome die Sensitivität der Melanomfrüherkennung. jekt zudem mit dem Förderpreis der Niedersächsischen Krebsgesellschaft e.V. ausgezeichnet. Arbeitshypothese der bis dato andauernden Studie war, dass Pigmentläsionen in der beginnenden malignen Transformation mehr dynamische Änderungen aufweisen als benigne atypische Nävi. Die Erfassung dynamischer Änderungen (wie z.B. eines asymmetrischen Flächenwachstums) von klinisch atypischen aber nicht melanomverdächtigen Nävi durch sequentielle Speicherung von Verlaufsbildern sollte es demnach ermöglichen, initiale maligne Melanome besser zu identifizieren [1].

\section{Patienten und Methoden \\ $\nabla$}

Gemäß der zuletzt erfolgten Datenanalyse wurden im Rahmen der Studie 530 Patienten (43,5\% weibliche, 56,5\% männliche) anhand von Anzahl/ Phänotyp der Nävi und der Eigen- und Familienanamnese für Melanome in Risikogruppen einge- 


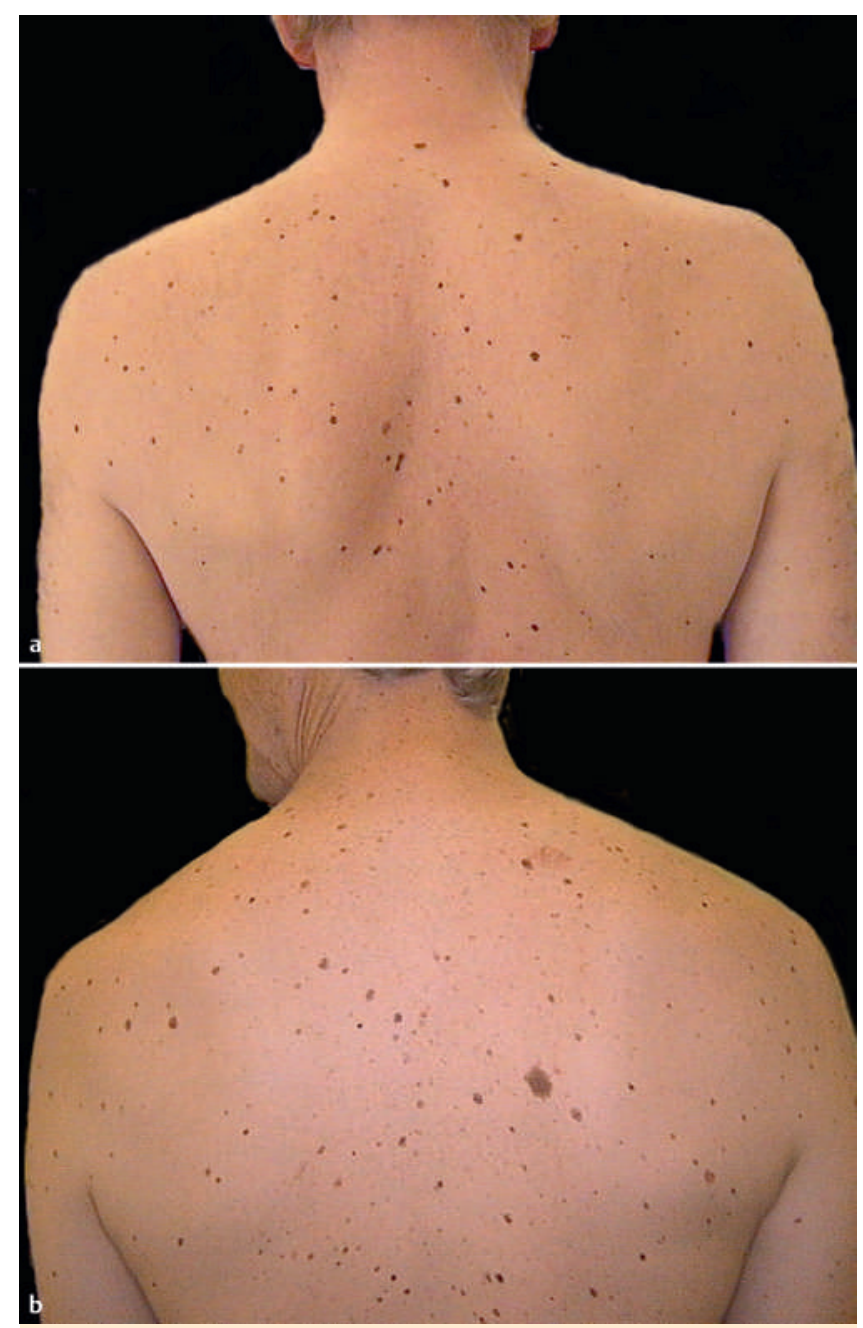

Abb. 1 Übersichtsaufnahmen von zwei Patienten der Studie. Aufgrund der Vielzahl an atypischen Nävi besteht ein erhöhtes Risiko an einem Melanom zu erkranken.

teilt und in regelmäßigen Abständen (alle 3-12 Monate) klinisch, auflichtmikroskopisch und digital-auflichtmikroskopisch untersucht. Die Risikogruppe I (MN=multiple Nävi) umfasste Patienten mit $\geq 50$ Nävi insgesamt, die Risikogruppe II (DN = dysplastische Nävi) Patienten mit $\geq 50$ Nävi und $\geq 3$ atypischen Nävi und die Risikogruppe III (FAMMM-Syndrom = familiäres atypisches „multiple mole melanoma“) Patienten, welche die Kriterien der Gruppe II erfüllten und $\geq 2$ Melanome in der Familienanamnese aufwiesen.

Der durchschnittliche Beobachtungszeitraum der Patienten betrugt 32,2 Monate. Es wurden 7001 atypische, aber nicht melanomsuspekte Pigmentläsionen in regelmäßigen Intervallen digital-auflichtmikroskopisch überwacht. Für die Definition einer klinisch bzw. auflichtmikroskopisch erkennbaren Atypie wurden von Pehamberger et al. und Stolz et al. formulierte Kriterien, wie die alleinige Asymmetrie oder eine alleinige unregelmäßige Pigmentierung herangezogen $[2,3]$.

Die Patientenuntersuchung erfolgte für die Studie in drei voneinander getrennten Durchgängen. Der erste Untersuchungsdurchgang erfasste die Krankheitsvorgeschichte (z. B. sich verändernde Pigmentmale, Bluten eines Pigmentmales) und die Untersuchung mit dem bloßen Auge. Im zweiten Durchgang wurden die Patienten mit einem konventionellen Auflichtmikroskop untersucht. Im dritten Durchgang erfolgte eine digitale Erfas-

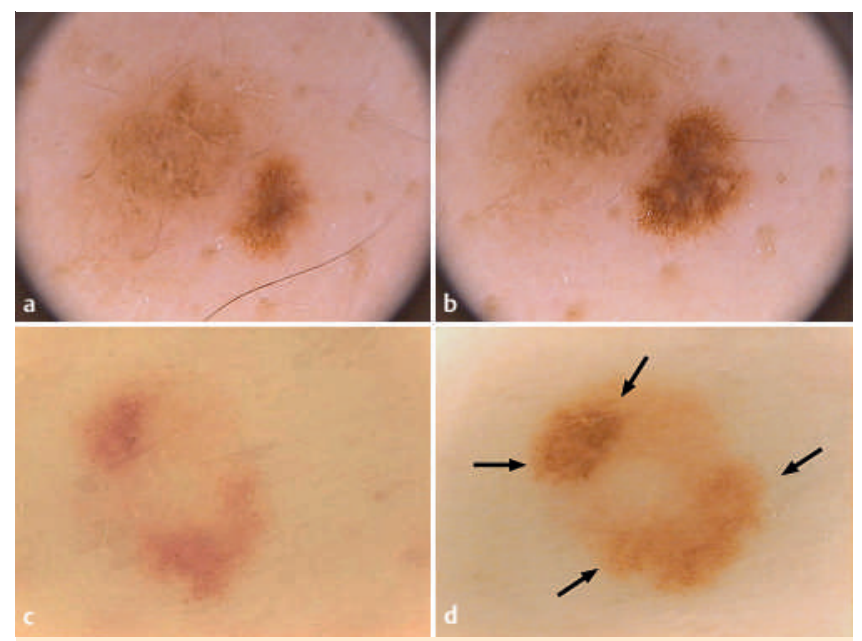

Abb. 2 Verlaufsveränderungen bei zwei atypischen Pigmentläsionen in der digitalen Dermatoskopie: Die erste Läsion (a) besteht aus zwei getrennten pigmentierten Arealen. Der im Bild rechte Anteil ist intensiv pigmentiert und zeigt im Verlauf ein symmetrisches Größenwachstum (b). Beobachtungsintervall: 6 Monate. Histologische Beurteilung: Melanoma in situ. Die zweite Läsion ist unregelmäßig pigmentiert (c) und zeigt im Verlauf von ebenfalls 6 Monaten ein symmetrisches Größenwachstum sowie eine fokale Zunahme der Pigmentierung (Pfeile in (d)). Histologische Beurteilung: Invasives superfiziell spreitendes Melanom, Tumordicke nach Breslow 0,25 mm.

sung und Speicherung zuvor festgelegter atypischer Pigmentmale. Die gespeicherten Bilder wurden bei den Folgeterminen mit den korrespondierenden Voraufnahmen verglichen, um dynamische Veränderungen der atypischen Pigmentmale zu erfassen. Für jede exzidierte Läsion wurde der entscheidende Untersuchungsgang (bloßes Auge vs. konventionelle Auflichtmikroskopie vs. digitale Verlaufsbilder) prospektiv erfasst.

Läsionen, welche gemäß eines etablierten Algorithmus für Auflichtmikroskopie (7 Point Ckecklist [4]) „melanomsuspekt“ waren, wurden umgehend exzidiert und nicht einer weiteren Beobachtung zugeführt. Digital gespeicherte atypische Pigment-Läsionen mit Größenwachstum jeglicher Art, Änderung der Pigmentstruktur oder Auftreten von Malignitätskriterien im Verlauf, wurden ebenfalls exzidiert. Eine Exzision wurde in diesen Fällen auch dann vorgenommen, wenn die konventionelle Auflichtmikroskopie keinen Melanomverdacht ergab.

\section{Ergebnisse \\ $\nabla$}

Zum Zeitpunkt der Erstvorstellung wurden 237 Pigmentläsionen exzidiert. Darunter befanden sich 37-maligne Melanome (Erfolgsrate: 1 Melanom auf 6 Exzisionen).

Während des Beobachtungszeitraumes konnten 53 Melanome (25 invasive Melanome, 28 in situ Melanoma) unter 637 exzidierten Pigmentläsionen identifiziert werden (Erfolgsrate: $1 \mathrm{Me}-$ lanom auf 12 Exzisionen). 18 dieser Melanome wurden ausschließlich aufgrund der Analyse der digitalen auflichtmikroskopischen Verlaufsbilder entdeckt (Erfolgsrate: 1 Melanom auf 19 Exzisionen). In diesen Fällen wurden geringgradige Verlaufsveränderungen der atypischen Nävi festgestellt, ohne dass nach Analyse mittels etablierter diagnostischer Algorithmen für Auflichtmikroskopie der Verdacht für ein Melanom vorlag ( $\bullet$ Abb.2). Die sequentielle digitale Dermatoskopie ermöglicht somit durch die Identifizierung zusätzlicher Melanome die Stei- 


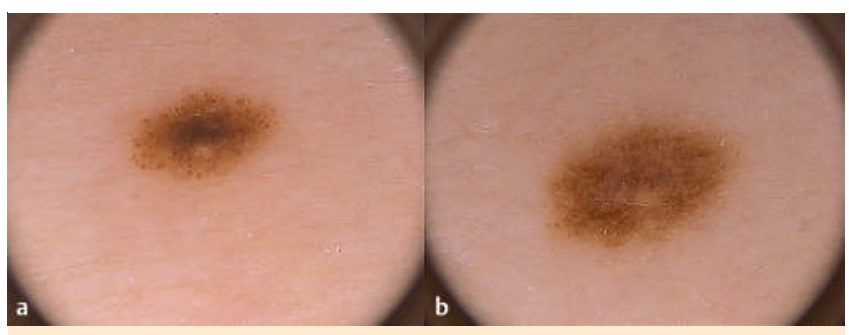

Abb. 3 Verlaufsveränderungen bei einer atypischen Pigmentläsion in der digitalen Dermatoskopie: Die Läsion zeigt eine zentral intensive Pigmentierung und kleine, gleichmäßig im Randbereich verstreute globuläre Strukturen (sog. Randglobules [a]). Im Verlauf von 12 Monaten wächst die Läsion stark symmetrisch und ist nun weitgehend homogen pigmentiert (b). Histologische Beurteilung: Dysplastischer Compound Nävus.

gerung der Sensitivität der konventionellen Auflichtmikroskopie. Dies gilt insbesondere für Patienten mit stark erhöhtem Melanomrisiko (Dysplastisches Nävussyndrom, FAMMM Syndrom). Im Rahmen der Studie fand sich eine signifikante Korrelation zwischen dem Auftreten von Melanomen und der zugeteilten Risikoklasse (I-III). Bei den Einzelfaktoren fand sich eine signifikante Korrelation zwischen dem Melanomerkrankungsrisiko während der Studie und der Anzahl der Nävi bzw. der Eigen-/ Familienanamnese für ein malignes Melanom. Andere bekannte Risikofaktoren wie Haarfarbe, Augenfarbe, Hauttyp oder Epheliden ergaben keine signifikante Korrelation zum Auftreten eines malignen Melanoms während der Studie.

Die 18-malignen Melanome, welche ausschließlich aufgrund der digitalen Verlaufsuntersuchung detektiert wurden, zeigten im Rahmen der Studie die geringste mittlere Tumordicke nach Breslow von 0,31 mm (mittlere Tumordicke für Melanome die durch konventionelle Auflichtmikroskopie detektiert wurden: $0,53 \mathrm{~mm})$.

\section{Kommentar}

Die digitale sequentielle Dermatoskopie und der Vergleich der gespeicherten Bilder mit den Ausgangsbefunden (bei Erstvorstellungstermin) ermöglichte die Detektion von 18 zusätzlichen Melanomen, welche durch die konventionelle Dermatoskopie zu diesem Zeitpunkt nicht auffällig waren. Somit wurden im Beobachtungszeitraum 34\% der detektierten Melanome (18 von 53) allein aufgrund des digitalen Bildvergleichs erkannt. Die digitale sequentielle Dermatoskopie steigerte daher durch die Detektion zusätzlicher Melanome die Sensitivität der Melanom-Früherkennung.

Die Patienten mit dem höchsten Melanomrisiko profitierten am meisten von der digitalen Technik. In der Risikogruppe III (FAMMM-Syndrom) wurden 66,7\% der Melanome allein durch diese Technik identifiziert, in der Risikogruppe II (dysplastische Nävi) immerhin noch 32,5\% der Melanome.

\section{Fallstricke der digitalen sequentiellen Dermatoskopie}

\section{Hoher Zeitaufwand}

Die in der Studie angewandte Untersuchungstechnik ist sehr zeitaufwendig. Für eine Erstvorstellung mit Anfertigung der digitalen Übersichtsaufnahmen werden ca. 30 Arbeitsminuten be- nötigt (in Extremfällen, bei FAMMM Patienten bis $60 \mathrm{~min}$ ), für jede Folgeuntersuchung müssen ca. 20 Arbeitsminuten veranschlagt werden.

\section{Noch niedrige Spezifität in der Melanomerkennung}

Die Erfolgsrate des alleinigen digitalen Bildvergleiches mit Detektion von 1 Melanom unter 19 Exzisionen verdeutlicht die noch niedrige Spezifität der Technik. Dies liegt zum Großteil an der weit gefassten Indikationsstellung zur Exzision digital gespeicherter Läsionen in der Studie (jegliche Größenzunahme, jegliche Änderung der Pigmentierung, usw.). Eine wichtige Zukunftsaufgabe besteht in der Erarbeitung eines Algorithmus zur Steigerung der Spezifität bei erhaltener hoher Sensitivität. So ist gemäß unserer Daten ein alleiniges geringes symmetrisches Flächenwachstum $(<10 \%$ in einem Jahr) sehr selten ein Zeichen einer malignen Transformation, war jedoch das häufigste Kriterium, das im Rahmen der Studie zur Exzision aufgrund des digitalen Verlaufes führte ( $\boldsymbol{O}$ Abb. $\mathbf{3})$.

\section{Auswahl der Läsionen für digitale Beobachtung}

Die größte Schwierigkeit der digitalen sequentiellen Dermatoskopie liegt in der richtigen Auswahl von Läsionen, welche tatsächlich für eine weitere Beobachtung geeignet sind. Auf keinen Fall dürfen melanomsuspekte Läsionen gewählt werden. Eine Beobachtung solcher Läsionen würde die operative Therapie verzögern und die Prognose dieser Patienten durch eine gesteigerte Tumordicke nach Breslow verschlechtern.

Um tatsächlich die Chance auf die Detektion zusätzlicher Melanome zu eröffnen, sollten ausschließlich Pigmentläsionen mit Zeichen der klinischen Atypie (z. B. alleinige Asymmetrie bei homogener Pigmentierung) für eine weitere Beobachtung ausgewählt werden.

\section{Der Leitspruch: IF IN DOUBT, CUT IT OUT bleibt weiterhin gültig.}

\section{Abstract}

\section{Early Detection of Melanoma Through Digital Epiluminescence Microscopy (DELM) $\nabla$}

Patients with a high number of atypical nevi and a personal and/ or family history of melanoma are at high risk to develop cutaneous melanoma. During the course of a specially designed documentation and surveillance programme using epiluminescence microscopy (ELM) and digital epiluminescence microscopy (DELM) high-risk patients $(n=530)$ were categorized by the number and phenotype of their nevi and their personal and family history of melanoma. Patients were screened in 3-12 months intervals by the unaided eye, ELM, and DELM. Median follow-up was 32.2 months. Examinations at the first visit identified $37 \mathrm{cu}-$ taneous melanomas. During the following observation period, another 53 melanomas were identified. Eighteen of these 53 melanomas were exclusively identified based upon DELM criteria. In these cases, subtle lesional changes occurred over time, and ELM diagnostic algorithms for differentiating benign melanocytic lesions from melanoma did not score a suspicion of melanoma. All melanomas, either pre-existing or developing during follow-up, were identified in an early, curable phase of tumour growth (Breslow $<1 \mathrm{~mm}$ ). We conclude that follow-up of patients at high risk for melanoma by a combination of naked-eye, dermoscopy, and especially digital dermoscopy allowed the early detection of melanomas. Sequential digital dermoscopy in- 
creased the sensitivity of melanoma detection by identifying additional melanomas.

\section{Literatur}

1 Haenssle HA, Krueger $U$, Vente $C$ et al. Results from an observational trial: digital epiluminescence microscopy follow-up of atypical nevi increases the sensitivity and the chance of success of conventional dermoscopy in detecting melanoma. J Invest Dermatol 2006; 126: 980-985
2 Pehamberger $H$, Binder $M$, Steiner A, Wolff $K$. In vivo epiluminescence microscopy: improvement of early diagnosis of melanoma. J Invest Dermatol 1993; 100: 356S-362S

3 Stolz W, Schiffner R, Pillet L et al. Improvement of monitoring of melanocytic skin lesions with the use of a computerized acquisition and surveillance unit with a skin surface microscopic television camera. J Am Acad Dermatol 1996; 35: 202 - 207

4 Argenziano G, Fabbrocini G, Carli P et al. Epiluminescence microscopy for the diagnosis of doubtful melanocytic skin lesions. Comparison of the $A B C D$ rule of dermatoscopy and a new 7-point checklist based on pattern analysis. Arch Dermatol 1998; 134: $1563-1570$ 